\title{
Characteristics and outcomes of diabetic patients with an implantable cardioverter defibrillator in a real world setting: results from the Israeli ICD registry
}

\author{
Hillel Steiner ${ }^{1,7^{*}}$, Michael Geist ${ }^{1}$, Ilan Goldenberg ${ }^{2}$, Mahmoud Suleiman ${ }^{3}$, Michael Glikson², \\ Alexander Tenenbaum ${ }^{2,4}$, Moshe Swissa ${ }^{5}$, Enrique Z. Fisman ${ }^{2,4}$, Gregory Golovchiner ${ }^{6}$, Boris Strasberg 6 , \\ Alon Barsheshet ${ }^{6}$ and On behalf of the Israeli Working Group of Pacing and EP
}

\begin{abstract}
Aims: There are limited data regarding the effect of diabetes mellitus (DM) on the risks of both appropriate and inappropriate implantable cardioverter defibrillator (ICD) therapy. The present study was designed to compare the outcome of appropriate and inappropriate ICD therapy in patients with or without DM.

Methods and results: The risk of a first appropriate ICD therapy for ventricular tachyarrhythmias (including anti tachycardia pacing and shock) was compared between 764 DM and 1346 non-DM patients enrolled in the national Israeli ICD registry. We also compared the risks of inappropriate ICD therapy, and death or cardiac hospitalization between diabetic and non-diabetic patients. Diabetic patients were older, were more likely to have ischemic cardiomyopathy, lower ejection fraction, atrial fibrillation, and other co-morbidities. The 3-year cumulative incidence of appropriate ICD therapy was similar in the DM and non-DM groups (12 and 13\%, respectively, $\mathrm{P}=0.983$ ). Multivariate analysis showed that DM did not affect the risk of appropriate ICD therapy ( $H R=1.07,95 \% \mathrm{Cl} 0.78-1.47, \mathrm{p}=0.694)$ or inappropriate therapy $(\mathrm{HR}=0.72,95 \% \mathrm{Cl} 0.42-1.23, \mathrm{p}=0.232)$. However, DM was associated with a $31 \%$ increased risk for death or cardiac hospitalization $(p=0.005)$. Results were similar in subgroup analyses including ICD and defibrillators with cardiac resynchronization therapy function recipients, primary or secondary prevention indication for an ICD.
\end{abstract}

Conclusions: Despite a significant excess of cardiac hospitalizations and mortality in the diabetic population, there was no difference in the rate of ICD treatments, suggesting that the outcome difference is not related to arrhythmias.

Keywords: Implantable cardioverter defibrillator, Diabetes mellitus, Heart failure, Outcomes

\section{Background}

Several randomized trials have shown that an implantable cardioverter-defibrillator (ICD) can improve survival both among patients who have had sustained ventricular tachyarrhythmias and among selected patients who have systolic heart failure (HF) without ventricular arrhythmia [1-3]. Patients with evidence of systolic HF with

\footnotetext{
*Correspondence: hillels@wmc.gov.il

${ }^{7}$ Department of Cardiology, The Edith Wolfson Medical Center, Holon, Israel

Full list of author information is available at the end of the article
}

intraventricular conduction delay may further benefit by implantation of a defibrillator with cardiac resynchronization function (CRTD) which may improve left ventricular function, prevent heart failure events and survival [4, 5] while obesity in mild heart failure did not diminish the clinical benefit of cardiac resynchronization therapy to reduce risk for appropriate ICD therapy [6].

Patients with diabetes mellitus (DM) are at increased risk for sudden cardiac death and heart failure [7-14]; a previous study suggests that diabetes mellitus may affect appropriate and inappropriate ICD discharge [14]. In addition, patients with obesity and overweight derived 
more benefit from CRT. Higher BMI was independently associated with better clinical outcome in CRT patients [15].

Importantly, little is known about the rates of ICD and CRTD therapies in DM patients as compared to non DM patients in a real life setting. Thus, we aimed to investigate whether DM may affect appropriate ICD therapy, inappropriate ICD therapy, HF hospitalization or death among patients enrolled in the Israel ICD registry.

\section{Methods}

\section{Study population}

The Israeli ICD Database is a prospective, national, multicenter registry of all patients implanted with an ICD or CRT-D for primary and secondary prevention in the 21 implanting centers of Israel $[16,17]$. The registry was initiated in July, 2010, and prospective follow up was started in July, 2011. At baseline, clinical and implantation characteristics were entered by the local electrophysiologist into a secure, web based electronic case report form. Follow up data for clinical and arrhythmic events were obtained from consecutively enrolled patients at 6 month intervals.

Patients were classified as having DM if they reported treatment for DM at the index hospitalization for device implantation or replacement or they were diagnosed with DM according to their medical chart. Other clinical variables collected included basic demographics, indication for implantation, electrocardiographic QRS morphology, left ventricular ejection fraction, New York Heart Association (NYHA) functional class, co-morbidities, and medications. The registry was approved by the institutional review board of each participating center and patients were included after providing written informed consent.

\section{Endpoints}

The endpoints evaluated at follow up included all-cause mortality and hospitalization for heart failure as well as device therapies that were further classified as being appropriate or inappropriate therapies including antitachycardia pacing (ATP) and ICD shock. All intracardiac electrograms of therapies were reviewed by the attending electrophysiologist who determined if the therapies were appropriate or not.

\section{Statistical methods}

Continuous variables were expressed as mean \pm standard deviation (SD). Categorical data were summarized as frequencies and percentages. Characteristics of patients categorized by diabetes status were compared by the Wilcoxon rank-sum test or Chi square test, as appropriate. The probabilities of appropriate ICD therapy, inappropriate ICD therapy and cardiac hospitalization or death by
DM status were graphically displayed according to the method of Kaplan and Meier, with comparison of cumulative events by the log-rank test.

The best subsets regression procedure was used to identify significant variables to be included in the multivariate regression models. For a model with the endpoint of death or cardiac hospitalization the variables included age, sex, ischemic heart disease, history of atrial fibrillation, NYHA functional class III-IV vs. I-II, creatinine level, and DM that was forced into the model. For a model with appropriate ICD therapy endpoint the variables included age, primary vs. secondary prevention indication, history of atrial fibrillation, QRS width, history of any ventricular arrhythmias (non-sustained or sustained VT), LVEF, device type (CRTD vs. ICD), and DM which was forced into the model. Analyses were conducted with SAS software (version 9.4, SAS institute, Cary, NC, USA). A 2 -sided $p$ value $<0.05$ was used for declaring statistical significance.

\section{Results}

From July 2010 through December 2014 a total of 2110 patients underwent ICD or CRTD implantations in 21 centers in Israel for whom follow up data was available. Patients were followed for a mean \pm SD of $21 \pm 10.2$ months.

We identified 764 (36\%) patients with DM and 1346 (64\%) without DM. The DM patients were significantly more ill in a wide variety of ways: they tended to be older, and had more co-morbid conditions, including atrial fibrillation, chronic lung disease, end stage renal failure on dialysis, hypertension, cerebrovascular disease, smoking and sleep apnea (Table 1). Diabetics were more likely to have more severe congestive HF, a wider QRS, a lower ejection fraction, to undergo CRTD implantation and to have ischemic cardiomyopathy.

DM patients implanted with a defibrillator (ICD or CRTD) had poor prognosis compared with non- diabetic patients implanted with a defibrillator.

At 3 years of follow up, the cumulative event rate of cardiac hospitalization or death was 38\% among DM patients and $29 \%$ among no-DM patients ( $\mathrm{p}<0.001$, Fig. 1). The cumulative event rate of death was 12 and $6 \%$ in DM and no-DM patients, respectively $(\mathrm{p}=0.021)$.

By multivariate analysis (Table 2), DM was associated with a significant $31 \%$ increased risk for death or cardiac hospitalization $(\mathrm{p}=0.005)$, and a trend towards 1.5 -fold increased risk for death $(\mathrm{p}=0.104)$. During study follow up 114 patients $(7 \%)$ with a primary prevention indication and 82 patients (16\%) with a secondary prevention indication received an appropriate ICD therapy. There were also 78 patients (4\%) who experienced an inappropriate ICD therapy during study follow up. However, 
Table 1 Patient characteristics

\begin{tabular}{lllc}
\hline & Non-diabetics (\%) & Diabetic (\%) & p value \\
\hline $\mathrm{N}$ & 1346 & 764 & \\
Male & $1215(82)$ & $726(85)$ & 0.033 \\
Age (mean \pm SD) & $62.2 \pm 14$ & $66.3 \pm 9.4$ & $<0.001$ \\
Age $\geq 75$ & $256(19)$ & $163(21)$ & 0.154 \\
PriorVA & $452(34)$ & $273(31)$ & 0.252 \\
Primary prevention & $901(66)$ & $589(76)$ & $<0.001$ \\
Ischemic heart disease & $904(67)$ & $625(82)$ & $<0.001$ \\
History of AF & $254(19)$ & $170(22)$ & $<0.001$ \\
Chronic lung disease & $85(6)$ & $118(16)$ & $<0.001$ \\
Smoker & $379(29)$ & $272(36)$ & 0.003 \\
Dialysis & $12(1)$ & $23(4)$ & $<0.001$ \\
Sleep apnea & $81(6)$ & $89(12)$ & $<0.001$ \\
Prior CVA & $98(7)$ & $67(9)$ & $<0.001$ \\
Atrial fibrillation & $30(2)$ & $35(1)$ & 0.05 \\
NYHA $\geq 3$ & $377(28)$ & $326(43)$ & $<0.001$ \\
EF & $30.5 \pm 11.6$ & $28.0 \pm 8.3$ & $<0.001$ \\
QRS duration & $115.8 \pm 29.8$ & $124.6 \pm 30.9$ & $<0.01$ \\
CRTD & $412(31)$ & $332(43)$ & $<0.001$ \\
ACE inhibitor & $921(69)$ & $603(79)$ & $<0.001$ \\
Beta Blocker & $1044(78)$ & $666(87)$ & $<0.001$ \\
\hline VAventricuar & & &
\end{tabular}

$V A$ ventricular arrhythmia, $C V A$ cerebrovascular accident, $A F$ atrial fibrillation, NYHA New York Heart Association, EF ejection fraction, CRTD cardiac resynchronization therapy-defibrillator, $A C E$ angiotensin converter enzyme

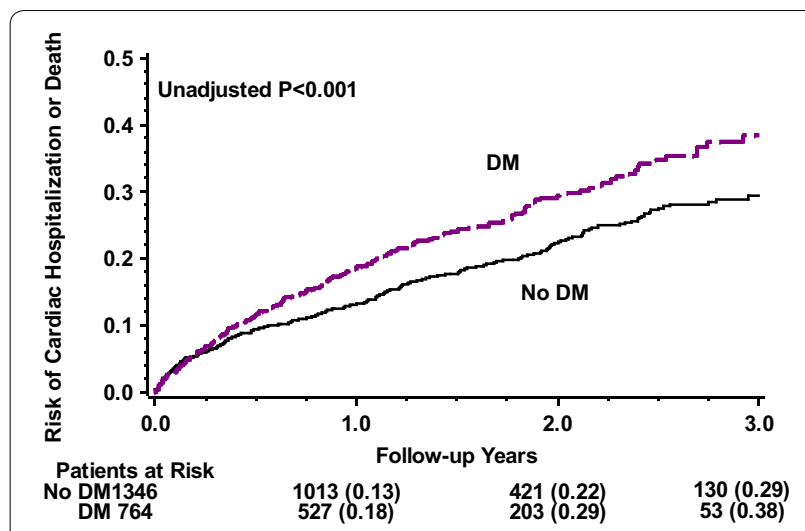

Fig. 1 Cumulative probability of cardiac hospitalization or death among diabetic and non-diabetic patients

Table 2 Multivariate analysis: diabetes mellitus and the risk of cardiac hospitalization or death

\begin{tabular}{llll}
\hline & Hazard ratio & $\mathbf{9 5 \%} \mathrm{Cl}$ & p value \\
\hline Cardiac hospitalization or death & 1.31 & $1.08-1.57$ & 0.005 \\
Death & 1.49 & $0.92-2.41$ & 0.104 \\
Cardiac hospitalization & 1.23 & $1.00-1.50$ & 0.047 \\
\hline
\end{tabular}

Adjusted for age, sex, ischemic heart disease, history of atrial fibrillation, NYHA functional class III-IV vs. I-II, and creatinine level there was no significant difference in the incidence of appropriate ICD therapy between the groups (Fig. 2). At 3 years of follow up the cumulative event rate of appropriate ICD therapy was $12 \%$ in the DM patients and $13 \%$ in the no-DM patients $(\mathrm{p}=0.983)$. Similarly, there was no significant difference in the incidence of inappropriate ICD therapy between the groups (Fig. 3) with 5\% among the DM patients and $6 \%$ among the non-DM patients ( $\mathrm{p}=0.075$, Fig. 2).

Consistent with these findings, multivariate analysis (Table 3) showed that DM did not affect the risk of appropriate ICD therapy [hazard ratio $(\mathrm{HR})=1.07, \mathrm{p}=0.694$ ], including appropriate shock $(\mathrm{HR}=1.13, \mathrm{p}=0.519)$ and appropriate ATP $(\mathrm{HR}=1.07, \mathrm{p}=0.743)$. $\mathrm{DM}$ also did not affect the risk of inappropriate ICD therapy $(H R=0.72$, $\mathrm{p}=0.232)$ including inappropriate shock $(\mathrm{HR}=0.67$, $\mathrm{p}=0.312)$, and inappropriate ATP $(0.82, \mathrm{p}=0.517$, Table 3).

Results were similar in subgroup analyses, demonstrating that DM was not associated with a significant

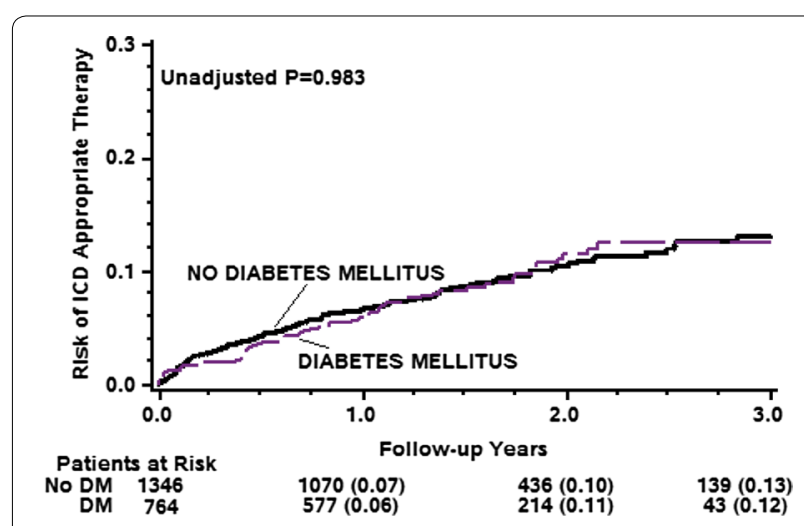

Fig. 2 Cumulative probability of appropriate ICD therapy among diabetic and non-diabetic patients

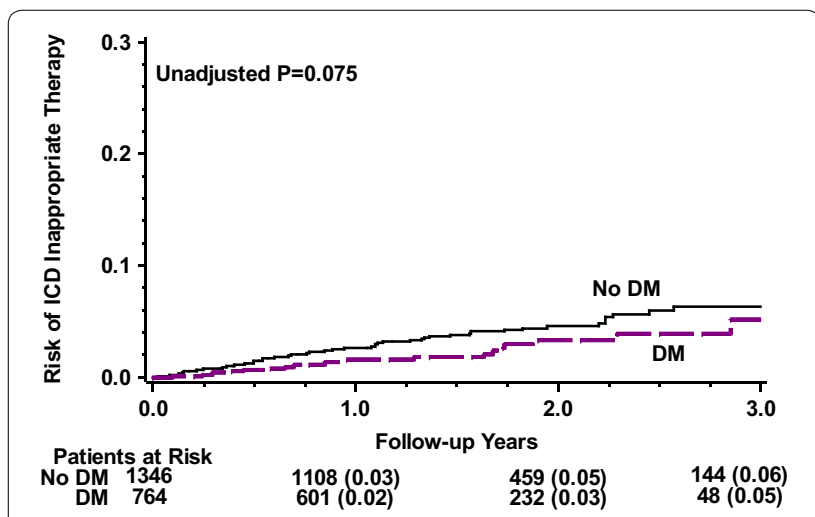

Fig. 3 Cumulative probability of inappropriate ICD therapy among diabetic and non-diabetic patients 
Table 3 Multivariate analysis: diabetes mellitus and the risk of appropriate and inappropriate ICD therapies

\begin{tabular}{llll}
\hline & Hazard ratio & $\mathbf{9 5 \%} \mathbf{C l}$ & p value \\
\hline Appropriate therapies* $^{*}$ & 1.07 & $0.78-1.47$ & 0.694 \\
Appropriate ATP & 1.13 & $0.79-1.62$ & 0.519 \\
Appropriate shock $^{\text {Inappropriate therapies }}{ }^{\#}$ & 1.07 & $0.70-1.64$ & 0.743 \\
Inappropriate ATP & 0.72 & $0.42-1.23$ & 0.232 \\
Inappropriate shock & 0.67 & $0.31-1.46$ & 0.312 \\
\hline
\end{tabular}

* Adjusted for age, primary vs. secondary prevention indication, history of atrial fibrillation, QRS width, history of any ventricular arrhythmias (non-sustained or sustained VT), LVEF, and CRTD

\# Adjusted for age, sex, primary vs. secondary prevention indication, history of atrial fibrillation, LVEF, NYHA functional class III-IV vs. I-II, and CRTD

increased risk for an appropriate therapy among ICD recipients $(\mathrm{HR}=1.04, \mathrm{p}=0.843)$ or among CRT-D recipients $(H R=1.14, p=0.636)$, among patients with a primary prevention $(H R=1.00, p=0.985)$ or among patients with a secondary prevention indication for an $\operatorname{ICD}(\mathrm{HR}=1.15, \mathrm{p}=0.572)$.

\section{Discussion}

The relationship between glucose metabolism abnormalities and atrial fibrillation [18-20] as well as several other types of rhythm disturbances is well established in both clinical and experimental models [21-24]. In this context, our findings have several important implications regarding the risk associated with DM in a "real world" setting among patients implanted with a defibrillator (ICD or CRTD). We have shown that (1) DM patients implanted with a defibrillator have a significantly higher risk for cardiovascular hospitalization or death compared with no-DM patients. (2) Despite the poorer prognosis among DM patients, there was no significant difference in the risk for appropriate or inappropriate ICD therapies compared with no-DM patients.

Overall, the DM patient group had a higher risk profile for poor outcomes, being older, having more comorbidities specifically pulmonary disease and renal failure, and more advanced heart failure. Thus it could be anticipated that they would have a worse prognosis. This finding is consistent with other studies of ICD patients who have found DM to be an independent risk factor for mortality [25-27].

In our study, DM was not associated with an increased rate of ICD therapies of any type- including appropriate or inappropriate shocks or anti tachycardia pacing. This is in concordance with the post hoc sub group analyses of the comparison of medical therapy, pacing, and defibrillation in heart failure (COMPANION) and multicenter automatic defibrillator implantation trial (MADIT)II trials that did not find an increased rate of appropriate therapies in the DM cohort [28, 29]. However, a recent sub-study of the multicenter automatic defibrillator implantation trial-reduce inappropriate therapy (MADIT RIT) did find that DM patients had a 58\% increased risk of appropriate therapy $(p=0.003)$, but a $46 \%$ decreased risk of inappropriate therapy $(\mathrm{p}=0.002)$ compared to no DM patients during a mean follow up of 17 months [14]. The authors suggested that the increased risk for appropriate ICD therapy might be explained by a reduced autonomic function or vulnerable myocardium resulting from ischemia and fibrosis in DM patients rendering the myocardium more prone to produce ventricular arrhythmias. The authors hypothesized that the lower risk of inappropriate therapy in DM patients observed in their study might be explained by a lower likelihood of DM patients experiencing exercise-induced sinus tachycardia or rapid ventricular response due to the fact that patients with diabetes mellitus may be more immobile and sedentary, and may have autonomic dysfunction or neuropathy.

The discrepancies between our findings and the MADIT RIT sub study, a primary prevention trial, may result from a different patient mix. Our registry included much more ischemic cardiomyopathy patients in both the diabetics and non-diabetics subgroups (87/67\%) than MADIT RIT (64/48\%). Our registry did include 30\% implanted for secondary prevention-with far more nondiabetics in the high arrhythmia risk secondary prevention group (Table 1). However, we performed a subgroup analysis showing that DM was not associated with a significant increased risk for an appropriate or inappropriate therapy both among patients with a primary prevention or among patients with a secondary prevention indication for an ICD.

Our findings indicate that although DM patients do have a poor prognosis, the excess in mortality or hospitalization for heart failure is not due to ventricular tachyarrhythmias. DM causes other forms of heart failure not amenable to device therapy such as heart failure with a normal ejection fraction, thus the ICD may not prevent mortality from this form of heart failure. Some studies have found DM to be a risk factor for sudden cardiac arrest $[8-11,30]$ which is predominantly due to ventricular arrhythmias [31]. DM was thought to cause repolarization abnormalities, predisposing to these arrhythmias [12]. This assumption needs reconsideration.

Our findings show that attempts to reduce the excess cardiac mortality in DM patients should not focus on the treatment of arrhythmia, but on other aspects of diabetic treatment. Although glycemic control in the past did not improve cardiovascular outcomes of diabetic patients [32], newer treatment modalities have been promising. Recent studies have shown that 
standard treatment of heart failure may be augmented by inhibitors of sodium-glucose cotransporter 2 and glucagon-like peptide 1 analogues, both of whom may reduce cardiac mortality of DM patients in randomized, controlled trials [33-35].

Our other finding was that there was no difference in the rate of inappropriate shocks between the two groups. $\mathrm{DM}$ is a risk factor for development of atrial fibrillation [7, 13, 36-38] and indeed, more of our patients had atrial fibrillation (Table 1), a major cause of inappropriate shocks, and it would be anticipated that the rate of inappropriate shocks would be increased. Evidence based programming of these devices has greatly decreased the rate of inappropriate therapies especially in diabetics [14] and indeed several centers contributing to the registry were participants in successful programming trials during the course of follow up, which may explain why there was no difference in the rate of inappropriate therapies $[14,39]$.

There other possible explanations for our findings. Current medical regimens have effectively reduced the rate of ICD therapies to the point that ICD implantation in non-ischemic cardiomyopathy is not beneficial, and this may be true in ischemic cardiomyopathy as well. However, a significant difference did remain in the other cardiovascular outcomes.

A limitation of our study is the designation of patients as diabetics based on self-reporting rather than laboratory results such as serum glucose or glycosylated hemoglobin (HbA1c) levels that constitute the criteria for the diagnosis of diabetes. Missing also is data regarding the length of time since diagnosis and the degree of glycemic control and non-cardiac organ failure. Some patients classified as non-diabetics at entry may have developed diabetes during the follow up period, however the study mean follow up of 22 months would not be expected to cause target organ damage.

In summary, based on the data from the Israeli ICD registry we conclude that DM patients implanted with ICDs have increased mortality and hospitalizations as compared to no DM patients, but a similar rate of appropriate and inappropriate therapies. Further attempts to reduce the excess mortality and morbidity associated with DM in heart disease should target other aspects of heart failure in this high risk population.

\footnotetext{
Abbreviations

ATP: anti-tachycardia pacing; CRTD: cardioverter-defibrillator with cardiac resynchronization therapy; COMPANION: comparison of medical therapy, pacing, and defibrillation in heart failure; DM: diabetes mellitus; HF: heart failure; ICD: implantable cardioverter-defibrillator; MADIT: multicenter automatic defibrillator implantation trial; MADIT RIT: multicenter automatic defibrillator implantation trial-reduce inappropriate therapy trial; NYHA: New York Heart Association; SD: standard deviation.
}

\section{Authors' contributions}

HS conceived the study, participated in the data collection and wrote the manuscript. AB conceived the study, performed the statistical analysis and wrote the manuscript. IG conceived the study and helped create the registry. $\mathrm{MGI}$ and MSu created the Israeli ICD Registry from which the data was taken, and reviewed the manuscript. MGe, MSW, GG and BS recruited subjects and reviewed the manuscript. EZF and AT reviewed the manuscript. All authors read and approved the final manuscript.

\section{Author details}

${ }^{1}$ The Edith Wolfson Medical Center, Holon, affiliated with the Sackler School of Medicine, Tel Aviv University, Tel Aviv, Israel. ${ }^{2}$ The Chaim Sheba Medical Center, Tel Hashomer, affiliated with the Sackler School of Medicine, Tel Aviv University, Tel Aviv, Israel. ${ }^{3}$ Rambam Medical Center, Haifa, Israel. ${ }^{4}$ Cardiovascular Diabetology Research Foundation, Holon, Israel. ${ }^{5}$ Kaplan Medical Center, Rehovot The Hebrew University, Jerusalem, Israel. ${ }^{6}$ Rabin Medical Center, Petah Tikva, Israel. ${ }^{7}$ Department of Cardiology, The Edith Wolfson Medical Center, Holon, Israel.

\section{Acknowledgements}

The authors acknowledge the contribution of the Boston Scientific Corporation enabling the establishment and maintenance of the Israel ICD Registry, and the support of the Cardiovascular Diabetology Research Foundation, Holon, Israel.

\section{Competing interests}

Alexander Tenenbaum and Enrique Z. Fisman-Editors-in-Chief of Cardiovascular Diabetology, Boris Strasberg-Advisory board for Medtronic Corporation, rest of the authors declare that they have no competing interests.

\section{Availability of data and material}

The datasets during and/or analyzed during the current study are available from the corresponding author on reasonable request.

\section{Ethics approval and consent to participate}

The protocol was approved by the institutional review board of each participating center. Written, informed consent was obtained from each participating individual.

\section{Funding}

The study was supported by an unrestricted grant from Boston Scientific to the Israeli Working Group on Pacing and Electrophysiology and the Israeli Association for Cardiovascular Trails.

Received: 29 September 2016 Accepted: 22 November 2016

Published online: 01 December 2016

\section{References}

1. Moss AJ, Hall WJ, Cannom DS, Daubert JP, Higgins SL, Klein H, Levine JH, Saksena S, Waldo AL, Wilber D, et al. Improved survival with an implanted defibrillator in patients with coronary disease at high risk for ventricular arrhythmia. Multicenter Automatic Defibrillator Implantation Trial Investigators. N Engl J Med. 1996;335(26):1933-40.

2. Moss AJ, Zareba W, Hall WJ, Klein H, Wilber DJ, Cannom DS, Daubert JP, Higgins SL, Brown MW, Andrews ML. Prophylactic implantation of a defibrillator in patients with myocardial infarction and reduced ejection fraction. N Engl J Med. 2002;346(12):877-83.

3. Bardy GH, Lee KL, Mark DB, Poole JE, Packer DL, Boineau R, Domanski M, Troutman C, Anderson J, Johnson G, et al. Amiodarone or an implantable cardioverter-defibrillator for congestive heart failure. N Engl I Med. 2005;352(3):225-37.

4. Moss AJ, Hall WJ, Cannom DS, Klein H, Brown MW, Daubert JP, Estes NA 3rd, Foster E, Greenberg H, Higgins SL, et al. Cardiac-resynchronization therapy for the prevention of heart-failure events. N Engl J Med. 2009;361(14):1329-38.

5. Goldenberg I, Kutyifa V, Klein HU, Cannom DS, Brown MW, Dan A, Daubert JP, Estes NA 3rd, Foster E, Greenberg H, et al. Survival with cardiac-resynchronization therapy in mild heart failure. N Engl I Med. 2014;370(18):1694-701. 
6. Szepietowska B, Polonsky B, Sherazi S, Biton Y, Kutyifa V, McNitt S, Aktas M, Moss AJ, Zareba W. Effect of obesity on the effectiveness of cardiac resynchronization to reduce the risk of first and recurrent ventricular tachyarrhythmia events. Cardiovasc Diabetol. 2016;15:93.

7. Aksnes TA, Schmieder RE, Kjeldsen SE, Ghani S, Hua TA, Julius S. Impact of new-onset diabetes mellitus on development of atrial fibrillation and heart failure in high-risk hypertension (from the VALUE Trial). Am J Cardiol. 2008;101(5):634-8.

8. Albert CM, Chae CU, Grodstein F, Rose LM, Rexrode KM, Ruskin JN, Stampfer MJ, Manson JE. Prospective study of sudden cardiac death among women in the United States. Circulation. 2003;107(16):2096-101.

9. Curb JD, Rodriguez BL, Burchfiel CM, Abbott RD, Chiu D, Yano K. Sudden death, impaired glucose tolerance, and diabetes in Japanese American men. Circulation. 1995;91(10):2591-5.

10. Jouven X, Desnos M, Guerot C, Ducimetiere P. Predicting sudden death in the population: the Paris Prospective Study I. Circulation. 1999;99(15):1978-83.

11. Jouven X, Lemaitre RN, Rea TD, Sotoodehnia N, Empana JP, Siscovick DS. Diabetes, glucose level, and risk of sudden cardiac death. Eur Heart J. 2005;26(20):2142-7

12. Nakou ES, Mavrakis H, Vardas PE. Are diabetic patients at increased risk of arrhythmias? Hellenic J Cardiol. 2012;53(5):335-9.

13. Nichols GA, Reinier K, Chugh SS. Independent contribution of diabetes to increased prevalence and incidence of atrial fibrillation. Diabetes Care. 2009;32(10):1851-6

14. Ruwald MH, Zareba W, Jons C, Zhang C, Ruwald AC, Olshansky B, McNitt S, Bloch Thomsen PE, Shoda M, Merkely B, et al. Influence of diabetes mellitus on inappropriate and appropriate implantable cardioverter-defibrillator therapy and mortality in the multicenter automatic defibrillator implantation trial-reduce inappropriate therapy (MADIT-RIT) Trial. Circulation. 2013;128(7):694-701

15. Cai C, Hua W, Ding LG, Wang J, Chen KP, Yang XW, Liu ZM, Zhang S. Association of body mass index with cardiac reverse remodeling and long-term outcome in advanced heart failure patients with cardiac resynchronization therapy. Circ J. 2014;78(12):2899-907.

16. Konstantino Y, Haim M, Boxer J, Goldenberg I, Feldman A, Michowitz Y, Glikson M, Suleiman M. Clinical outcomes of single-versus dual-chamber implantable cardioverter defibrillators: lessons from the Israeli ICD registry. J Cardiovasc Electrophysiol. 2016;27(6):718-23.

17. Sabbag A, Suleiman M, Laish-Farkash A, Samania N, Kazatsker M, Goldenberg I, Glikson M, Beinart R. Contemporary rates of appropriate shock therapy in patients who receive implantable device therapy in a real-world setting: from the Israeli ICD Registry. Heart Rhythm. 2015:12(12):2426-33.

18. Grundvold I, Bodegard J, Nilsson PM, Svennblad B, Johansson G, Ostgren CJ. Sundstrom J. Body weight and risk of atrial fibrillation in 7169 patients with newly diagnosed type 2 diabetes; an observational study. Cardiovasc Diabetol. 2015;14:5

19. January CT, Wann LS, Alpert JS, Calkins H, Cigarroa JE, Cleveland JC Jr, Conti JB, Ellinor PT, Ezekowitz MD, Field ME, et al. 2014 AHA/ACC/HRS guideline for the management of patients with atrial fibrillation: executive summary: a report of the American College of Cardiology/American Heart Association Task Force on practice guidelines and the Heart Rhythm Society. Circulation. 2014;130(23):2071-104.

20. Chang SH, Wu LS, Chiou MJ, Liu JR, Yu KH, Kuo CF, Wen MS, Chen WJ, Yeh YH, See LC. Association of metformin with lower atrial fibrillation risk among patients with type 2 diabetes mellitus: a population-based dynamic cohort and in vitro studies. Cardiovasc Diabetol. 2014;13:123.

21. Sanjuan R, Blasco ML, Martinez-Maicas H, Carbonell N, Minana G, Nunez J, Bodi V, Sanchis J. Acute myocardial infarction: high risk ventricular tachyarrhythmias and admission glucose level in patients with and without diabetes mellitus. Curr Diabetes Rev. 2011;7(2):126-34

22. Axelsen LN, Calloe K, Braunstein TH, Riemann M, Hofgaard JP, Liang B, Jensen CF, Olsen KB, Bartels ED, Baandrup U, et al. Diet-induced prediabetes slows cardiac conductance and promotes arrhythmogenesis. Cardiovasc Diabetol. 2015;14:87.
23. Barth AS, Tomaselli GF. Cardiac metabolism and arrhythmias. Circ Arrhythm Electrophysiol. 2009;2(3):327-35.

24. Soltysinska E, Speerschneider T, Winther SV, Thomsen MB. Sinoatrial node dysfunction induces cardiac arrhythmias in diabetic mice. Cardiovasc Diabetol. 2014:13:122

25. Bilchick KC, Stukenborg GJ, Kamath S, Cheng A. Prediction of mortality in clinical practice for medicare patients undergoing defibrillator implantation for primary prevention of sudden cardiac death. J Am Coll Cardiol. 2012:60(17):1647-55

26. Stein KM, Mittal S, Gilliam FR, Gilligan DM, Zhong Q, Kraus SM, Meyer TE. Predictors of early mortality in implantable cardioverter-defibrillator recipients. Europace. 2009;11(6):734-40.

27. van Rees JB, Borleffs CJ, van Welsenes $\mathrm{GH}$, van der Velde ET, Bax JJ, van Erven L, Putter H, van der Bom JG, Schalij MJ. Clinical prediction model for death prior to appropriate therapy in primary prevention implantable cardioverter defibrillator patients with ischaemic heart disease: the FADES risk score. Heart. 2012;98(11):872-7.

28. Saxon LA, Bristow MR, Boehmer J, Krueger S, Kass DA, De Marco T, Carson P, DiCarlo L, Feldman AM, Galle E, et al. Predictors of sudden cardiac death and appropriate shock in the comparison of medical therapy, pacing, and defibrillation in heart failure (COMPANION) Trial. Circulation. 2006;114(25):2766-72.

29. Singh JP, Hall WJ, McNitt S, Wang H, Daubert JP, Zareba W, Ruskin JN, Moss AJ. Factors influencing appropriate firing of the implanted defibrillator for ventricular tachycardia/fibrillation: findings from the multicenter automatic defibrillator implantation trial II (MADIT-II). J Am Coll Cardiol. 2005:46(9):1712-20.

30. Rea TD, Pearce RM, Raghunathan TE, Lemaitre RN, Sotoodehnia N, Jouven $X$, Siscovick DS. Incidence of out-of-hospital cardiac arrest. Am J Cardiol. 2004;93(12):1455-60

31. Watanabe E, Tanabe T, Osaka M, Chishaki A, Takase B, Niwano S, Watanabe I, Sugi K, Katoh T, Takayanagi K, et al. Sudden cardiac arrest recorded during Holter monitoring: prevalence, antecedent electrical events, and outcomes. Heart Rhythm. 2014;11(8):1418-25.

32. Holman RR, Paul SK, Bethel MA, Matthews DR, Neil HAW. 10-year follow-up of intensive glucose control in type 2 diabetes. N Engl J Med. 2008;359(15):1577-89.

33. Marso SP, Daniels GH, Brown-Frandsen K, Kristensen P, Mann JF, Nauck MA, Nissen SE, Pocock S, Poulter NR, Ravn LS, et al. Liraglutide and cardiovascular outcomes in type 2 diabetes. N Engl J Med. 2016:375(4):311-22.

34. Zinman B, Wanner C, Lachin JM, Fitchett D, Bluhmki E, Hantel S, Mattheus M, Devins T, Johansen OE, Woerle HJ, et al. Empagliflozin, cardiovascular outcomes, and mortality in type 2 diabetes. N Engl J Med. 2015:373(22):2117-28

35. Marso SP, Bain SC, Consoli A, Eliaschewitz FG, Jódar E, Leiter LA, Lingvay I, Rosenstock J, Seufert J, Warren ML, et al. Semaglutide and cardiovascular outcomes in patients with type 2 diabetes. N Engl J Med. 2016;375(19):1834-44.

36. Benjamin EJ, Levy D, Vaziri SM, D'Agostino RB, Belanger AJ, Wolf PA. Independent risk factors for atrial fibrillation in a population-based cohort. The Framingham Heart Study. JAMA. 1994;271(11):840-4

37. Krahn AD, Manfreda J, Tate RB, Mathewson FA, Cuddy TE. The natural history of atrial fibrillation: incidence, risk factors, and prognosis in the Manitoba Follow-Up Study. Am J Med. 1995;98(5):476-84.

38. Schnabel RB, Sullivan LM, Levy D, Pencina MJ, Massaro JM, D'Agostino RB Sr, Newton-Cheh C, Yamamoto JF, Magnani JW, Tadros TM, et al. Development of a risk score for atrial fibrillation (Framingham Heart Study): a community-based cohort study. Lancet. 2009;373(9665):739-45.

39. Auricchio A, Schloss EJ, Kurita T, Meijer A, Gerritse B, Zweibel S, AISmad FM, Leng CT, Sterns LD. Low inappropriate shock rates in patients with single- and dual/triple-chamber implantable cardioverter-defibrillators using a novel suite of detection algorithms: PainFree SST trial primary results. Heart Rhythm. 2015;12:926. 